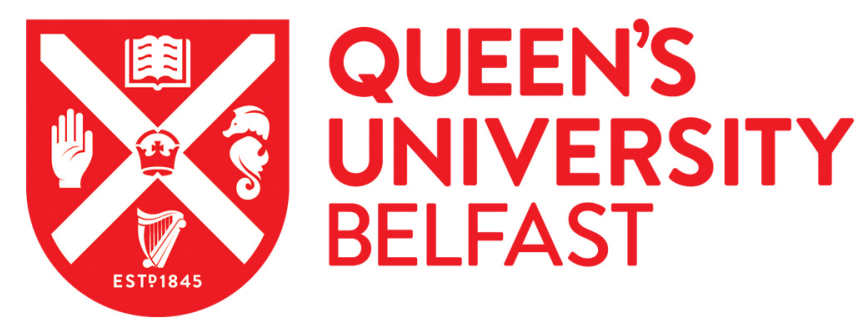

\title{
The design of a navigation guidance and control system for an unmanned surface vehicle for environmental monitoring
}

Naeem, W., Xu, T., Sutton, R., \& Tiano, A. (2008). The design of a navigation guidance and control system for an unmanned surface vehicle for environmental monitoring. Institution of Mechanical Engineers. Proceedings. Part M: Journal of Engineering for the Maritime Environment, 222(2), 67-79. https://doi.org/10.1243/14750902JEME80

Published in:

Institution of Mechanical Engineers. Proceedings. Part M: Journal of Engineering for the Maritime Environment

Queen's University Belfast - Research Portal:

Link to publication record in Queen's University Belfast Research Portal

\section{General rights}

Copyright for the publications made accessible via the Queen's University Belfast Research Portal is retained by the author(s) and / or other copyright owners and it is a condition of accessing these publications that users recognise and abide by the legal requirements associated with these rights.

Take down policy

The Research Portal is Queen's institutional repository that provides access to Queen's research output. Every effort has been made to ensure that content in the Research Portal does not infringe any person's rights, or applicable UK laws. If you discover content in the Research Portal that you believe breaches copyright or violates any law, please contact openaccess@qub.ac.uk. 


\section{Proceedings of the Institution of Mechanical Engineers, Part M: Journal of Engineering for the Maritime Environment \\ http://pim.sagepub.com/}

The design of a navigation, guidance, and control system for an unmanned surface vehicle for environmental monitoring

W Naeem, T Xu, R Sutton and A Tiano

Proceedings of the Institution of Mechanical Engineers, Part M: Journal of Engineering for the Maritime Environment 2008 222: 67

DOI: 10.1243/14750902JEME80

The online version of this article can be found at:

http://pim.sagepub.com/content/222/2/67

\section{Published by: \\ (-)SAGE \\ http://www.sagepublications.com \\ On behalf of: \\ Institution of \\ MiscintCA. \\ ENAाNत्रत्रक \\ Institution of Mechanical Engineers}

Additional services and information for Proceedings of the Institution of Mechanical Engineers, Part M: Journal of Engineering for the Maritime Environment can be found at:

Email Alerts: http://pim.sagepub.com/cgi/alerts

Subscriptions: http://pim.sagepub.com/subscriptions

Reprints: http://www.sagepub.com/journalsReprints.nav

Permissions: http://www.sagepub.com/journalsPermissions.nav

Citations: http://pim.sagepub.com/content/222/2/67.refs.html 


\title{
The design of a navigation, guidance, and control system for an unmanned surface vehicle for environmental monitoring
}

\author{
W Naeem ${ }^{1,2 *}, \mathbf{T ~ X u}^{1}, \mathbf{R}$ Sutton ${ }^{1}$, and A Tiano ${ }^{3}$ \\ ${ }^{1}$ School of Engineering, University of Plymouth, Plymouth, UK \\ ${ }^{2}$ School of Electronics, Electrical Engineering and Computer Science, Queen's University Belfast, Belfast, UK \\ ${ }^{3}$ Department of Information and Systems, University of Pavia, Italy
}

The manuscript was received on 22 January 2007 and was accepted after revision for publication on 27 July 2007.

DOI: 10.1243/14750902JEME80

\begin{abstract}
Maintaining the ecosystem is one of the main concerns in this modern age. With the fear of ever-increasing global warming, the UK is one of the key players to participate actively in taking measures to slow down at least its phenomenal rate. As an ingredient to this process, the Springer vehicle has been designed and is being developed for environmental monitoring and pollutant tracking. This paper highlights the Springer hardware and software architecture including various navigational sensors, a speed controller, and an environmental monitoring unit. In addition, details regarding the modelling of the vessel are outlined which are based mainly on recent trials data. The formulation of a fault tolerant multi-sensor data fusion technique is also presented. Moreover, control strategy based on a linear quadratic Gaussian controller is developed and simulated on the Springer model.
\end{abstract}

Keywords: unmanned surface vehicle, navigation, guidance and control, linear quadratic Gaussian, multi-sensor data fusion, pollutant tracking

\section{INTRODUCTION}

The potential of the use of unmanned surface vehicles (USVs) for tasks such as shallow-water surveying, weapon delivery, environmental data gathering, coordinating with autonomous underwater vehicles (AUVs), and surveillance is quite lucrative. Functionally, they are much simpler than AUVs and yet quite versatile for the kinds of mission that they are able to perform. In fact, the accuracy of USVs is far better than that of the AUVs because of the availability of global positioning system (GPS) fixes at all time in open waters. In contrast, AUVs regularly need to surface in order to correct for the error incurred by the dead-reckoning navigation.

Some well-known USV projects around the globe have been detailed in reference $[\mathbf{1}]$. The UK research interest in this key area has mainly been confined to

*Corresponding author: Now at: School of Electronics, Electrical Engineering and Computer Science, Ashby Buildings, Stranmillis Road, Queen's University Belfast, Belfast, BT9 5AH, UK that being undertaken by Corfield [2] who developed variants of Mimir EV1 for naval and surveying missions, and Young and Phillips [3] who developed a semisubmersible for deploying sensors for ocean surveys. Of late, Reed et al. [4] from the US Naval Academy have published the design of a small monohull autonomous surface vehicle whilst Hook [5] enlisted his findings on the existing USV types that are known to have recently been or are being developed.

Also mentioned in the list is the Springer USV which has been designed and is being developed at the University of Plymouth, UK. Springer is intended to be a cost-effective and environmentally friendly USV which is designed primarily for undertaking pollutant tracking, and environmental and hydrographic surveys in rivers, reservoirs, inland waterways, and coastal waters, particularly where shallow waters prevail. An equally important secondary role is also envisaged for Springer as a test bed platform for other academic and scientific institutions involved in environmental data gathering, sensor 
and instrumentation technology, control systems engineering, and power systems based on alternative energy sources.

In order for the vehicle to be capable of undertaking the kinds of mission that are contemplated, Springer requires a robust, reliable, accurate, and adaptable navigation, guidance, and control (NGC) system which allows seamless switching between automatic and manual control modes. Whilst AUVs are now in service in the offshore industry, such craft cannot be deployed in shallow or inland waters to perform the kind of tasks outlined above. As a result, operational costs are currently high, as scuba divers or special vessels containing a number of personnel have to be employed. It is foreseen that Springer will be portable and capable of operating in water from 1 to $60 \mathrm{~m}$ in depth.

Several articles have already been published by the present authors regarding the development of Springer USV (see, for example, references [1], [6], and [7]). This paper covers some of the previously published material and amalgamates it with up-todate developments that have been carried out on the vessel. This mainly includes the trial results from experiments conducted at Roadford Reservoir, Devon, UK. A model of the catamaran is deduced from the trials data using system identification (SI) techniques and presented in section 3. Section 4 details the Springer NGC architecture. This includes the formulation and simulation of a control algorithm based on linear quadratic Gaussian (LQG) theory for the identified model. In addition, a faulttolerant navigation subsystem based on a fuzzy multisensor data fusion methodology is also elaborated.

\section{SPRINGER HARDWARE-SOFTWARE ARCHITECTURE}

The Springer USV was designed as a medium waterplane twin-hull vessel which is versatile in terms of mission profile and payload. It is approximately $4 \mathrm{~m}$ long and $2.3 \mathrm{~m}$ wide with a displacement of $0.6 \mathrm{t}$. A schematic diagram is shown in Fig. 1 showing the layout of the components within the hulls where each hull is divided into three watertight compartments.

The data acquisition (DAQ) and NGC systems are carried within watertight Peli cases that are placed in a bay area between the cross-beams. This facilitates the quick substitution of systems on shore or at water. This is illustrated in Fig. 2(a) showing both

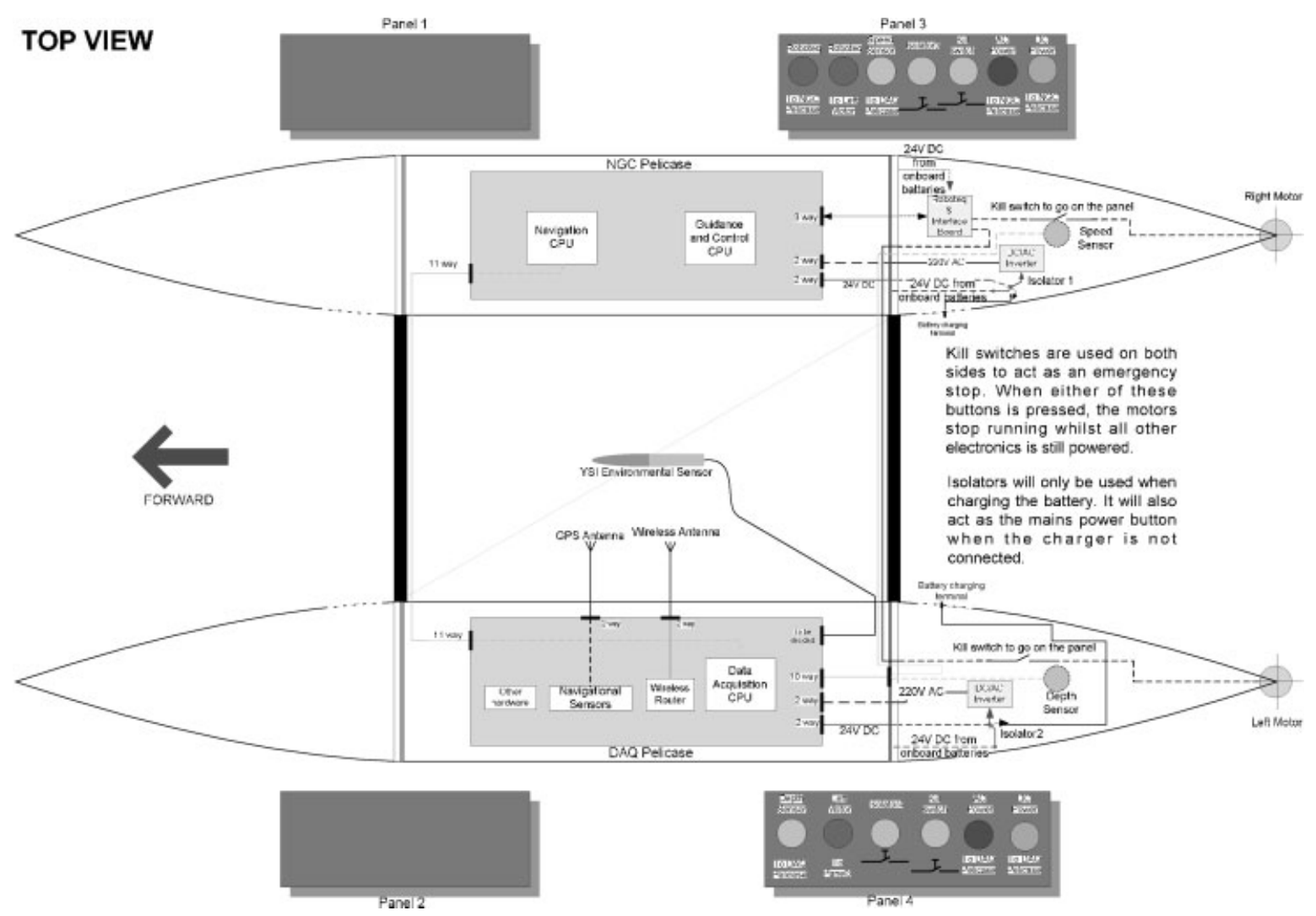

Fig. 1 A schematic layout of Springer vehicle showing all onboard components (CPU, central processing unit) 


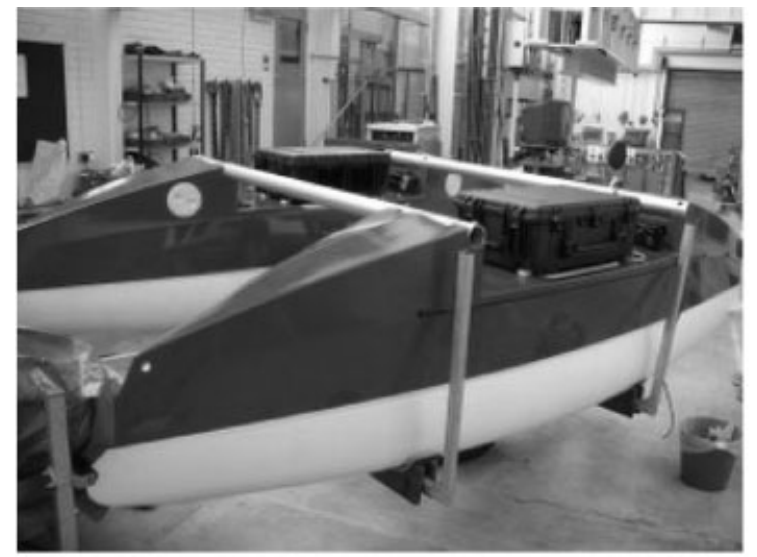

(a)

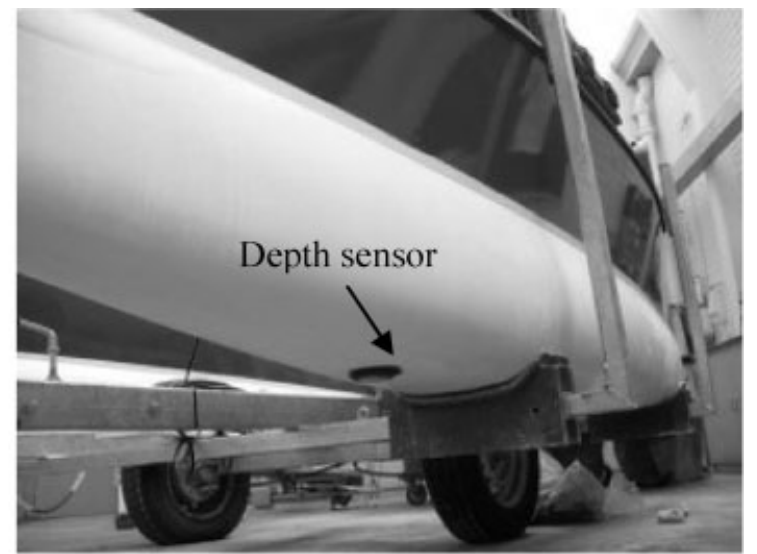

(b)

Fig. 2 (a) Springer's USV showing clearly the Peli cases in the bay area between the crossbeams. (b) Speed sensor installed at the bottom of the vehicle in the rear section

Peli cases. In order to maintain the temperature inside the Peli cases, an economical but effective cooling system based on heat sinks is installed. This is vital for the onboard sensors; otherwise the heat generated by the onboard computers could gradually build up to a level well outside the operating temperature of the sensors. An experiment is carried out with and without the heat sinks in the Peli cases and it has been found that the heat sinks facilitate the regulation of temperature within the Peli cases.

With the exception of the depth and speed sensors that are located at the bottom of the hulls, all other sensors are placed within the Peli cases. The depth sensor is shown in Fig. 2(b) whilst the speed sensor is located at the bottom of the rear section of the other hull. Within the rear section of the hulls are present the motor controller, radio control systems, and a.c. power source for the onboard computers.
These are mounted on custom-made plates which are simple to install and replace.

The link from onboard electronics to the Peli cases is created through front panels, shown in Fig. 3(a), which are especially designed to accommodate various connectors, cable glands, isolators, and emergency kill switches. The purpose of the isolators is to separate the battery terminals from the electronics circuitry whilst being recharged whereas the emergency kill switches are installed on both panels and are manually operated from either side in case of an emergency. Another wired link is established between the DAQ and NGC personal computers (PCs) through a serial-ethernet cable laid across the hulls through the cross-beam.

The electronics and the propulsion system are powered by four $12 \mathrm{~V}$ gel technology batteries that are placed in each hull accessed by a watertight

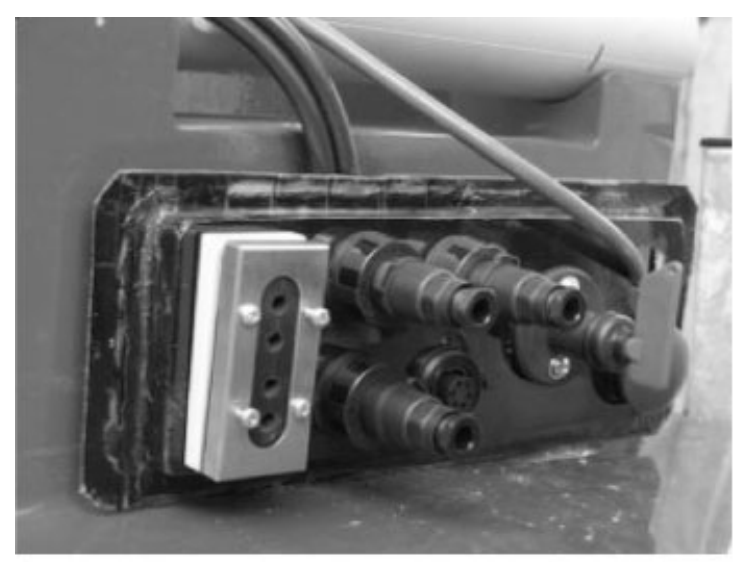

(a)

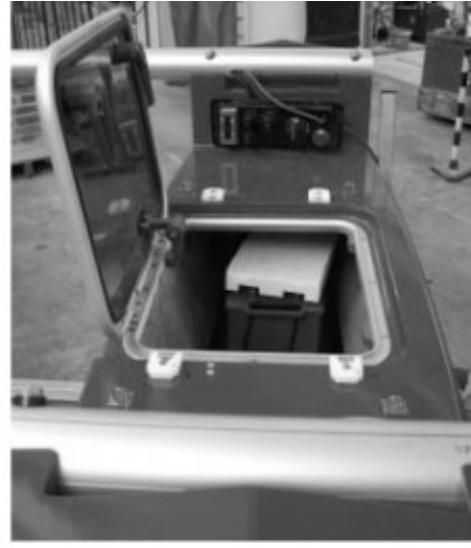

(b)

Fig. 3 (a) One of the front panels showing the connectors, the cable gland, the isolator, and an emergency switch. (b) The layout of the components including the batteries, hatch, and front panel is shown on one of the hulls 


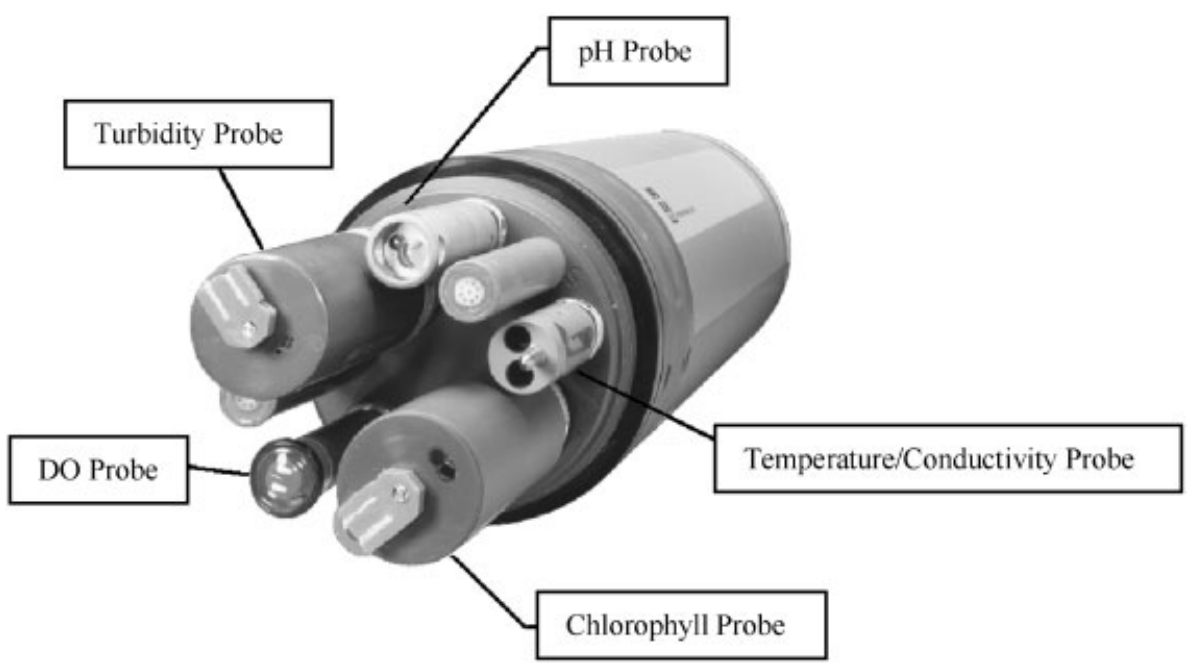

Fig. 4 A YSI environmental monitoring unit showing the locations of various sensors [8] (DO, dissolved oxygen)

hatch, as shown in Fig. 3(b). These are paired together to supply $24 \mathrm{~V}$ where each battery is capable of sourcing $135 \mathrm{~A}$ h of current.

In order to prevent any catastrophe resulting from a water leakage, leak sensors are utilized within the motor housing. If a breach is detected, the onboard computer immediately issues warning to the user and/or takes appropriate action in order to minimize damage to the onboard electronics.

A sensor that plays the vital role in carrying out Springer missions is the YSI environmental monitoring unit depicted in Fig. 4. This will be installed in the centre of the vehicle as illustrated in Fig. 1 on a purpose-built platform that could be lowered or raised depending upon the situation. This unit is capable of measuring several important parameters such as the $\mathrm{pH}$, dissolved oxygen, and turbidity, as shown in Table 1, and is vital in determining the water pollution level and hence the direction of the polluting source.

Furthermore, a mast is installed to carry the GPS and wireless antennae. The wireless antenna is used as a means of communication between the vessel and its user and is intended to be utilized for remote monitoring purposes, for intervention in the case of erratic behaviour, and to alter the mission parameters.
In order to minimize the noise pollution and to eradicate diesel fuel the Springer propulsion system consists of two propellers powered by a set of $24 \mathrm{~V}$ $74 \mathrm{lb}$ Minn Kota Riptide transom mount saltwater trolling motors which are placed in the rear of each hull. Steering of the vessel is based on differential propeller revolution rates. More will be said about the steering aspect in the guidance and control section. Details are now provided regarding the navigation suite, the speed controller, and the software architecture of the vessel.

\subsection{Navigation sensor suite}

In Springer, the integrated sensor suite combines a GPS, three different types of compass, a speed log, and a depth sensor. All these sensors are interfaced to a PC via an NI-PCI 8430/8 (RS232) serial connector. The navigation sensor suite is shown in a block diagram form in Fig. 5.

The redundancy in the compasses is a requirement of the navigation system which combines all data readings and provides the best estimate using a novel form of multi-sensor data fusion (MSDF) algorithm. The proposed MSDF strategy takes advantage of soft computing methodology to

Table 1 The YSI 6600 Sonde environmental monitoring unit specifications

\begin{tabular}{ll}
\hline Available sensors & $\begin{array}{l}\text { Temperature, conductivity, dissolved oxygen, pH, oxygen reduction potential, ammonium } \\
\text { nitrate, chloride, depth (shallow, medium, deep, or shallow vented), turbidity, chlorophyll, and } \\
\text { rhodamine WT }\end{array}$ \\
Operating temperature $\left({ }^{\circ} \mathrm{C}\right)$ & -5 to 45 \\
Operating depth $(\mathrm{m})$ & $0-200$ \\
Mass $(\mathrm{kg})$ & 3.18 \\
Interface & $\mathrm{RS}-232, \mathrm{SDI}-12$ \\
Power & $8 \mathrm{C}-$-size alkaline batteries or external $12 \mathrm{~V}$ d.c. \\
Battery life & Approximately 90 days at $20{ }^{\circ} \mathrm{C}$ at 15 min logging intervals \\
\hline
\end{tabular}




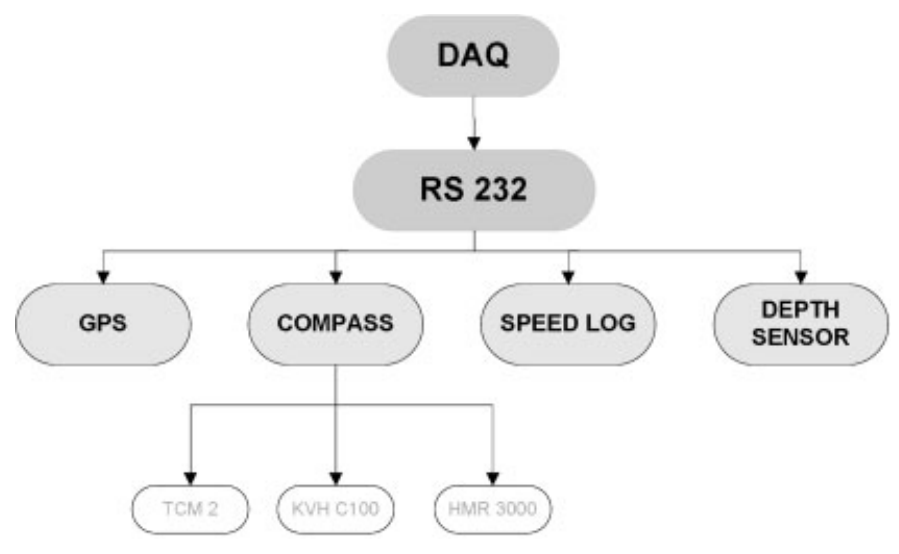

Fig. 5 Springer sensor suite

adapt to always present sensors' noise. This will be further elaborated in section 4 .

\subsection{RoboteQ controller}

To control the speed of the propulsion motors and hence the speed of the vessel, a two-channel RoboteQ AX2850 is installed in the vehicle. The AX2850 is a highly configurable microcomputerbased dual-channel digital speed controller which can accept speed commands in a variety of ways such as pulse-width-based control from a standard radio control receiver, analogue voltage commands, or RS-232 commands from a microcontroller or wireless modem. For Springer, the commands to the controller are sent using the serial port communication from the onboard guidance and control PC.

The controller's two channels can be operated independently or can be combined to set the forward-reverse direction and steering of the vehicle by coordinating the motion on each side of the vehicle. This can be operated in either open-loop or closed-loop mode. In closed-loop operation, actual speed measurements from tachometers or optical encoders are used to verify that the motor is rotating at the desired speed and to adjust the power to the motors accordingly [9].

\subsection{Software architecture}

Besides all the measurement sensors, the Springer is equipped with three onboard PCs running Windows XP. They are contained in the two Peli cases with a serial/ethernet link connecting them together. These machines are termed the DAQ PC, the navigation PC, and the control PC. The DAQ PC acquires data from all the sensors and transmits them over the serialethernet cable to the navigation and control PCs by concatenating the desired data in one string. The transmit rate from the DAQ $\mathrm{PC}$ is the actual sampling rate to be used in the software to tune the navigation and controller parameters that can be specified by the user. The navigation PC provides estimates of the states of the vehicle by combining data using a fault-tolerant MSDF technique described in section 3.1. The controller PC issues commands directly to the speed controller which produces a differential thrust (if needed) and steers the vehicle on the desired course.

The DAQ PC runs Labview where a user interface is created to monitor sensor readings and to alter the mission parameters if required. This user interface is accessible on a remote laptop through the wireless connection. The navigation and control PCs are running MATLAB software and are interfaced together using a serial link. As mentioned earlier, another role envisaged for the Springer is to be utilized by other marine research groups as an easy-to-use platform to test their own NGC algorithms. The use of MATLAB is an example of the ease of developing the NGC software to be directly implemented on Springer which offers an attractive feature to the marine research groups where MATLAB is generally used for system design and simulation.

The next section details the SI procedure and its application to the Springer vehicle.

\section{SYSTEM IDENTIFICATION AND MODELLING}

SI techniques have been applied to obtain the Springer model and hence controllers are developed subsequently. For this, several sea trials were carried out where the vessel was driven for some calculated manoeuvres and data recorded. A similar approach has been adopted in reference $[\mathbf{1 0}]$ to extract the 
model of an AUV and proved to be quite successful. An LQG controller will then be designed for the extracted model and tested in real time.

A block diagram of the complete SI procedure is depicted in Fig. 6, where $\boldsymbol{u}$ is the input, $\boldsymbol{y}$ is the output or response, $\boldsymbol{d}$ is the disturbance, $\hat{\boldsymbol{y}}$ is the response of the model to the same input $\boldsymbol{u}$ and $\varepsilon$ is the error between the model output and plant output also called the residuals. The objective of identification is to minimize the sum-squared errors or residuals $\varepsilon$. The interested reader is referred to reference [11] for a comprehensive treatment of this subject.

The next section applies SI to the Springer USV data obtained from recent trials.

\subsection{Application to the Springer USV}

In this section, the SI procedure is applied to data acquired from the Springer vehicle during recent experiments at Roadford Reservoir. The vehicle has a differential steering mechanism and thus requires two inputs to adjust its course. This can be simply modelled as a two-input single-output system in the form depicted in Fig. 7, where $n_{1}$ and $n_{2}$ are the two propeller thrusts in revolutions per minute. Clearly, straight-line manoeuvres require both the thrusters to run at the same speed whereas the differential thrust is zero in this case. In order to linearize the model at an operating point, it is assumed that the vehicle is running at a constant speed of 3 knots. This corresponds to both thrusters running at

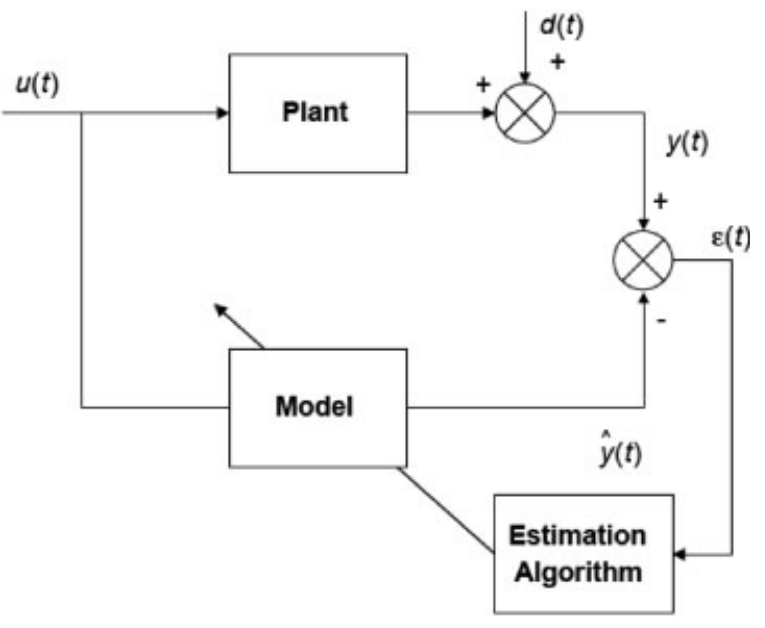

Fig. 6 The overall SI procedure

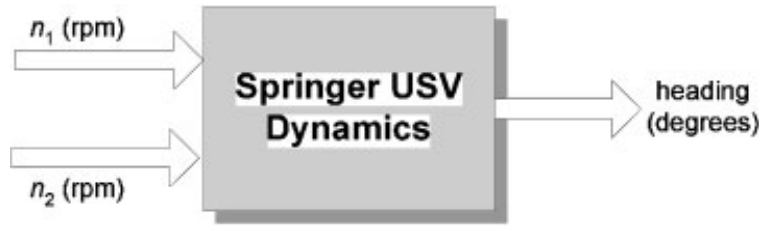

Fig. 7 Block diagram representation of a two-input USV

$900 \mathrm{r} / \mathrm{min}$. To clarify this further, let $n_{\mathrm{c}}$ and $n_{\mathrm{d}}$ represent the common mode and differential mode thruster velocities defined to be

$$
\begin{aligned}
& n_{\mathrm{c}}=\frac{n_{1}+n_{2}}{2} \\
& n_{\mathrm{d}}=\frac{n_{1}-n_{2}}{2}
\end{aligned}
$$

In order to maintain the velocity of the vessel, $n_{\mathrm{c}}$ must remain constant at all times. The differential mode input, however, oscillates about zero depending on the direction of the manoeuvre. For data acquisition, several inputs including a pseudorandom binary sequence was applied to the thrusters and the heading response was recorded. Figures 8(a) and (b) depict two data sets obtained from those trials. The input shown is the differential mode thruster velocity $n_{\mathrm{d}}$, which causes the vehicle to manoeuvre as required. The acquired data were processed and downsampled to $1 \mathrm{~Hz}$ since this frequency was deemed to be adequate for controller design.

SI was then applied to the acquired data set and a dynamic model of the vehicle is obtained using a prediction error method in the form

$$
\boldsymbol{y}(\boldsymbol{z})=G_{1}(\boldsymbol{z}) \boldsymbol{u}_{1}+G_{2}(\boldsymbol{z}) \boldsymbol{u}_{2}
$$

where $G_{1}$ and $G_{2}$ denote the discrete transfer functions from inputs $\boldsymbol{u}_{1}$ and $\boldsymbol{u}_{2}$ respectively and where $y$ is the output of the system. In this case, only $n_{\mathrm{d}}$ has been manipulated and therefore acts as the sole input to the system. This alters both $n_{1}$ and $n_{2}$ whereas $n_{\mathrm{c}}$ is maintained to conserve the operating regime. Two models of second and fourth order were identified from the data; however, a subsequent simulation study reveals that there is no significant advantage in using a more complex fourth-order model. Hence, the second-order model in state space form is selected for further analysis and controller design according to 

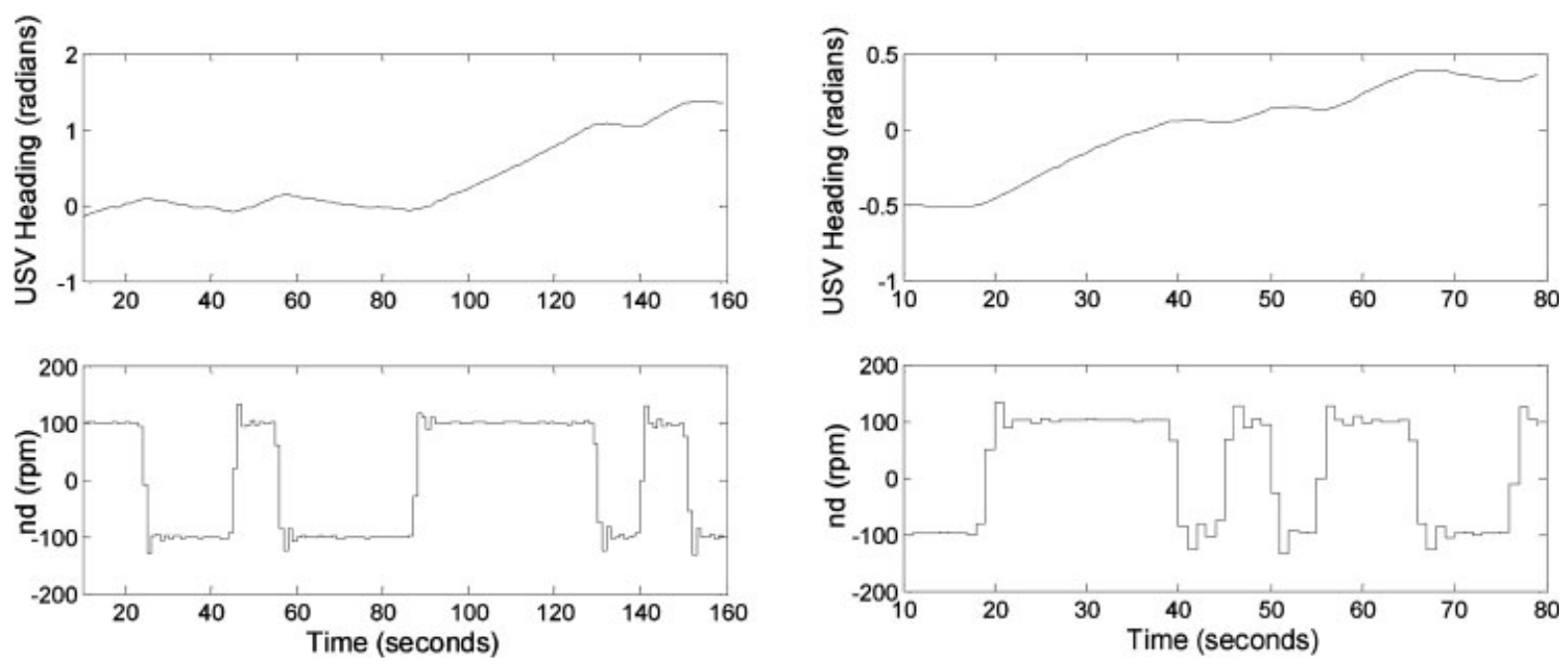

(a)

(b)

Fig. 8 Experimental data sets from trials conducted at Roadford Reservoir in Devon

$$
\begin{aligned}
& \boldsymbol{x}(k+1)=\mathbf{A} \boldsymbol{x}(k)+\mathbf{B} \boldsymbol{u}(k) \\
& \boldsymbol{y}(k)=\mathbf{C} \boldsymbol{x}(k)+\mathbf{D} \boldsymbol{u}(k)
\end{aligned}
$$

where

$$
\begin{aligned}
& \mathbf{A}=\left[\begin{array}{cc}
1.002 & 0 \\
0 & 0.9945
\end{array}\right], \mathbf{B}=\left[\begin{array}{c}
6.354 \times 10^{-6} \\
-4.699 \times 10^{-6}
\end{array}\right] \\
& \mathbf{C}=\left[\begin{array}{ll}
34.13 & 15.11
\end{array}\right], \quad \mathbf{D}=[0]
\end{aligned}
$$

See Appendix 1 for the notation. To validate the model, a cross-validation test and residual analysis are carried out. Figure 9(a) depicts the cross-validation test, which clearly shows the accuracy of the model compared with the measured response. Correlation analysis also reveals that the model is able to capture the dynamics of interest of the USV as shown in Fig. 9(b) since both autocorrelation and cross-correlation coefficients are within the confidence intervals.

An integrated NGC system is now developed for the identified model and is explained in the following section.
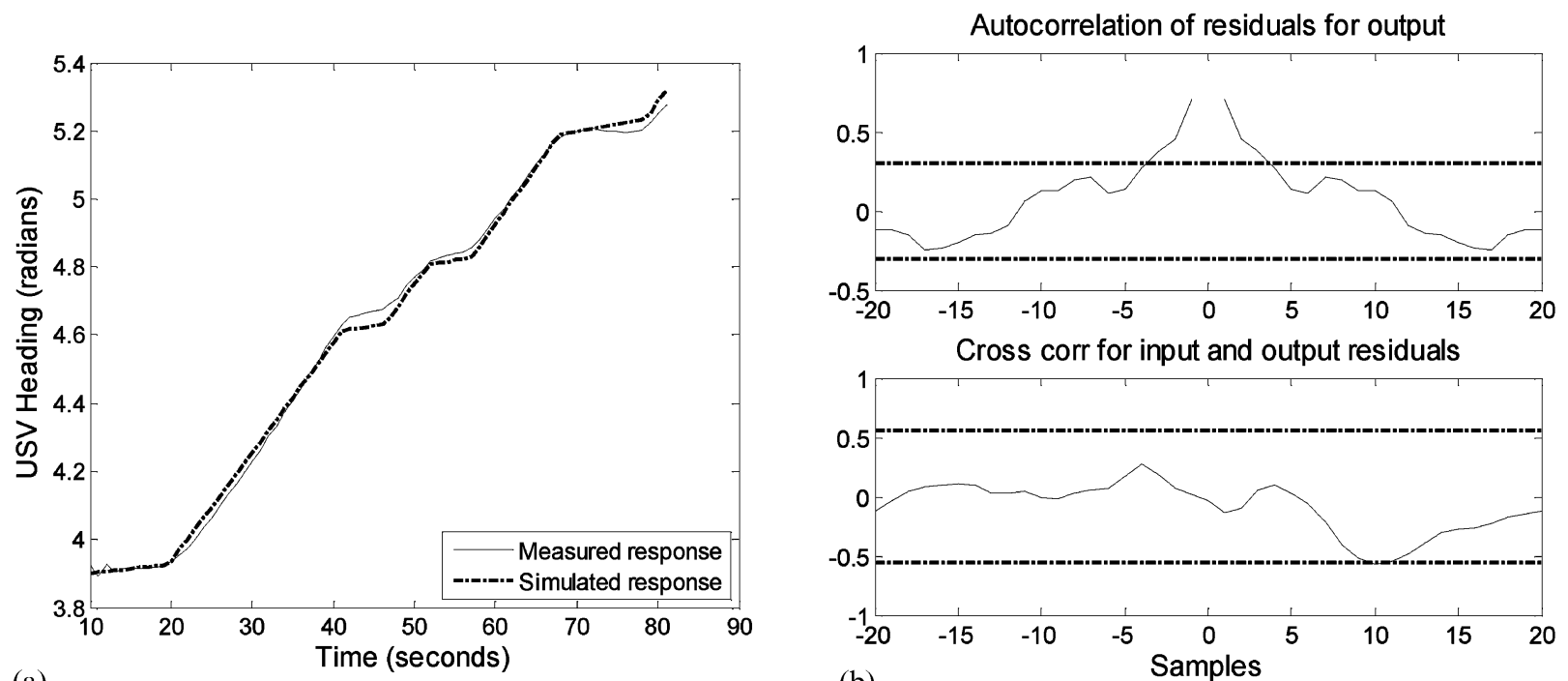

(a)

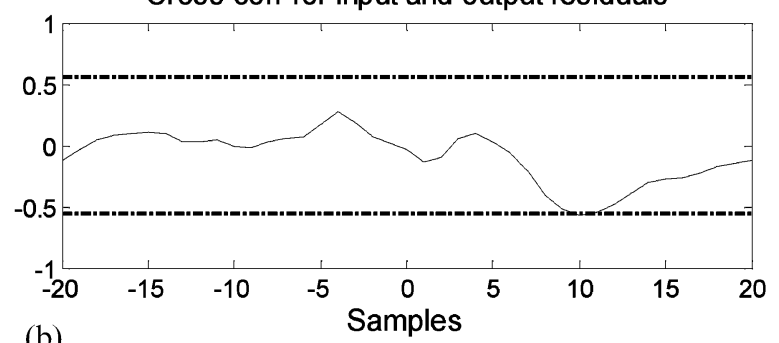

(b)

Fig. 9 (a) Actual measurements and simulated output of the USV model. (b) Cross-correlation and autocorrelation of the residuals 


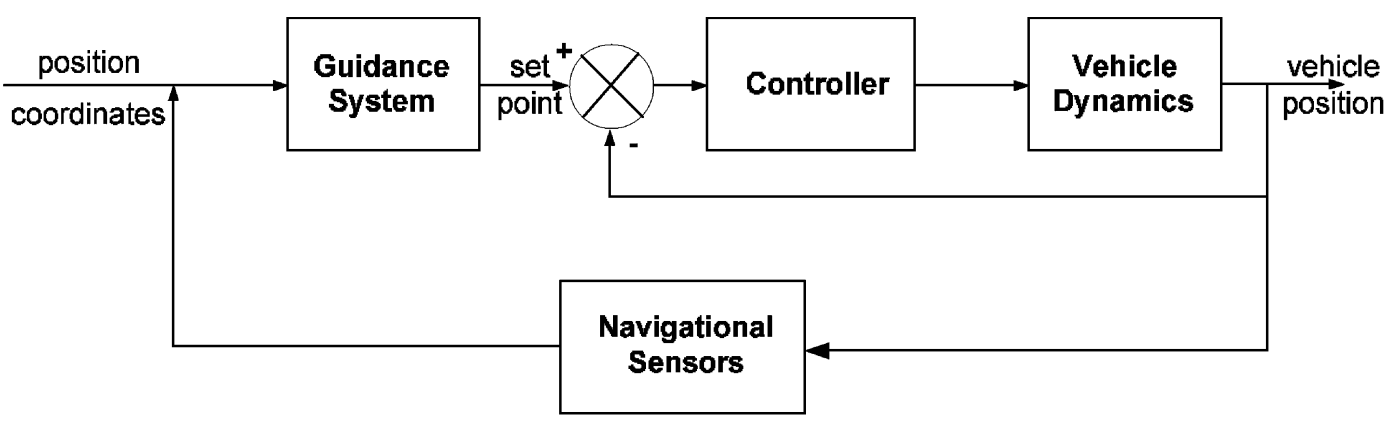

Fig. 10 NGC system of a vehicle

\section{NAVIGATION, GUIDANCE, AND CONTROL}

All autonomous vehicles must have onboard NGC systems, which should work in accord with each other for proper operation. Imperfections in one system degrade the efficiency of the other. The navigation system provides information related to the target, which is processed by the guidance system to generate reference headings. The control system is responsible for keeping the vehicle on course as specified by the guidance processor. A generic block diagram of an NGC system of a vehicle is depicted in Fig. 10.

\subsection{Fault-tolerant multi-sensor navigation strategy}

Navigation is defined as determining accurately the position and velocity of a moving vehicle relative to a known reference and to plan and execute the manoeuvres necessary to move between the desired locations [12]. Autonomous navigation means that a vehicle can move to a desired destination or along a desired path purposefully without human intervention. A good navigation system gathers information from every available source which is measured by different sensors. Various sensors provide their own distinguished outputs to provide a navigation solution. In many applications, more than one sensor is involved so as not only to determine the navigation states at a certain time but also to supply a continuous navigation trajectory. The term 'multisensor navigation system' is therefore often used. Such systems are typically operated with multiple sensors referenced to a common platform and synchronized to a common time base. Each sensor contributes its own stream of data and all the data are optimally processed.

It has been proven that multiple motion sensors play a vital role in autonomous navigation. In real situations, there is always the possibility of having sensor failure. Hence, to realize reliable and robust navigation in Springer, fault detection and isolation are the main concerns. In any of the sensor fault situations, the navigation system must immediately identify the failed sensor and act in such a way that data from the failed sensor do not corrupt the global estimates. A possible line of action is to isolate the sensor from the list of active sensors.

Owing to the capability to deal with complex problems, fuzzy logic adaptive (FLA) MSDF techniques have become the most popular approach for multi-sensor navigation. By using fuzzy logic, the uncertainty in sensor readings can be directly represented in the fusion process by allowing each proposition to be assigned a real number to indicate its degree of truth.

Based on the above discussion, a modified FLA federated Kalman filter (FKF)-based MSDF architecture is proposed in Fig. 11 to realize fault-tolerant multi-sensor navigation for Springer. The FLA FKF is a two-stage data-processing technique which divides the standard Kalman filter into $N$ local filters and a master filter. In the first stage, all local filters process their own data in parallel, according to the difference between the actual value and theoretical value of covariance $[\mathbf{1 3}]$, to yield the best possible local estimate. Fuzzy logic is then implemented to alter the measurement noise covariance matrix in order to decrease the sensor fault influence. Next, the feedback factors $\beta(i)$ are determined from the master filter to each local filter. These are generated online depending on the accuracy of each local Kalman filter estimate. The most accurate local Kalman filter receives the highest feedback from the master filter and thus makes the largest contribution in the global estimation process. Having done that, the master filter fuses all the local estimates to generate the best global solution.

A typical procedure for an FKF can be described mathematically as

$$
\mathbf{W}_{i}(k)=\sqrt[1]{\beta_{i}} \mathbf{W}_{f}(k)
$$




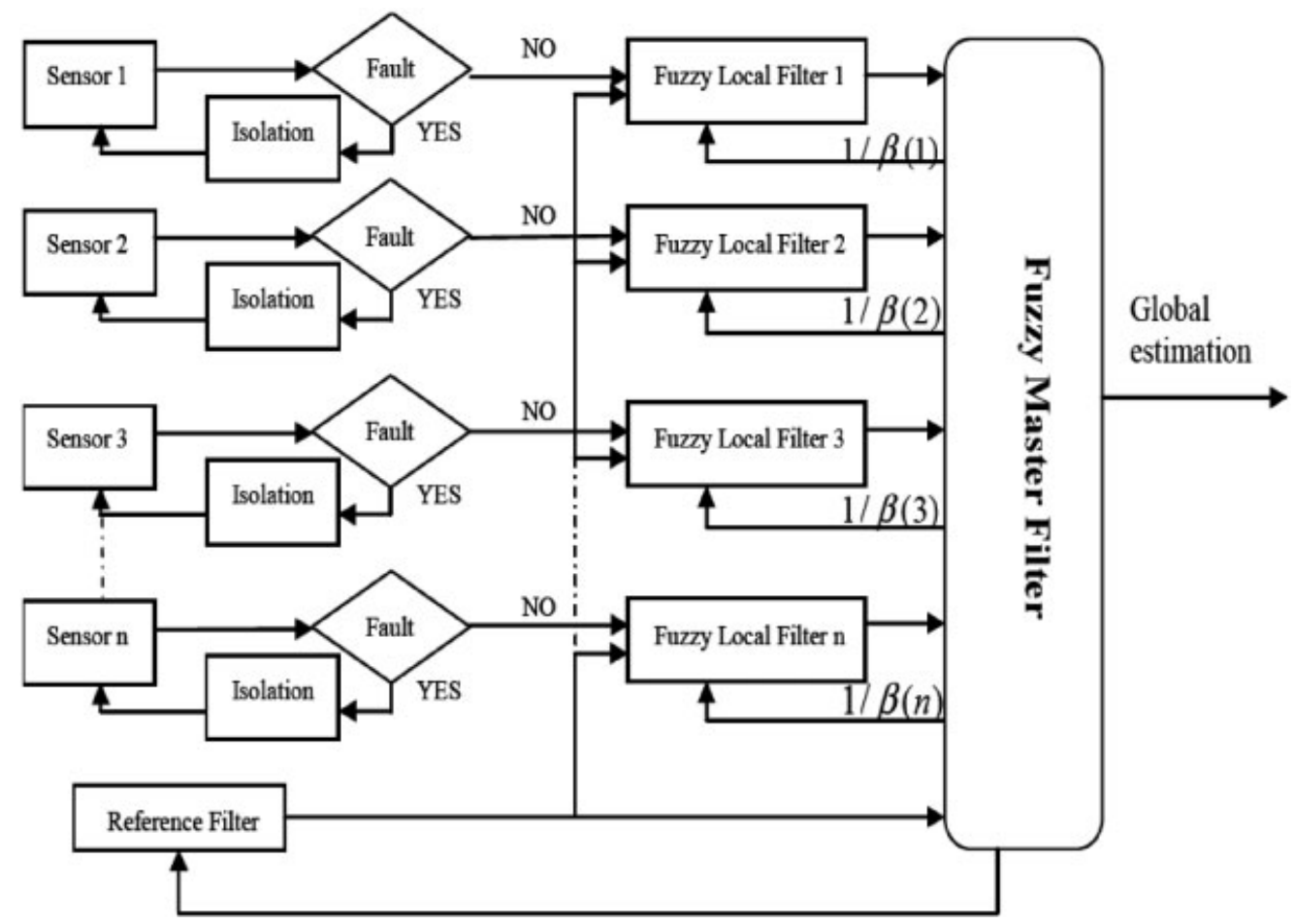

Fig. 11 MSDF strategy with a fault-tolerant feature

$$
\begin{aligned}
& \mathbf{P}_{i}^{+}(k)=\sqrt[1]{\beta_{i}} \mathbf{P}_{f}^{+}(k) \\
& \widehat{\boldsymbol{x}}_{i}^{+}(k)=\sqrt[1]{\beta_{i}} \widehat{\boldsymbol{x}}_{f}^{+}(k)
\end{aligned}
$$

where $i$ is the index of local Kalman filters, $i=1, \ldots, N$. In the local Kalman filters

$$
\hat{\boldsymbol{x}}_{i}^{+}(k)=\hat{\boldsymbol{x}}_{f}^{+}(k)
$$

subject to

$$
\begin{aligned}
& \sum_{i=1}^{N} \beta_{i}=1 \\
& \widehat{\boldsymbol{x}}_{i}^{-}(k+1)=\mathbf{A}_{i}(k) \widehat{\boldsymbol{x}}_{i}^{+}(k)+\mathbf{B}_{i}(k) \boldsymbol{u}_{i}(k) \\
& \mathbf{P}_{i}^{-}(k+1)=\mathbf{A}_{i}(k) \mathbf{P}_{i}^{+}(k) \mathbf{A}_{i}^{\mathrm{T}}(k)+\mathbf{W}_{i}(k) \\
& \mathbf{K}_{i}(k)=\mathbf{P}_{i}^{-}(k) \mathbf{C}_{i}^{\mathrm{T}}(k)+\left[\mathbf{C}_{i}(k) \mathbf{P}_{i}^{-}(k) \mathbf{C}_{i}^{\mathrm{T}}(k)+\mathbf{V}_{i}(k)\right]^{-1} \\
& \widehat{\boldsymbol{x}}_{i}^{+}(k)=\widehat{\boldsymbol{x}}_{i}^{-}(k)+\mathbf{K}_{i}(k)\left[z_{i}(k)-\mathbf{C}_{i}(k) \widehat{\boldsymbol{x}}_{i}^{-}(k)\right]
\end{aligned}
$$

$$
\begin{aligned}
\mathbf{P}_{i}^{+}(k)= & {\left[\mathbf{I}-\mathbf{K}_{i}(k) \mathbf{C}_{i}(k)\right] \mathbf{P}_{i}^{-1}(k)\left[\mathbf{I}-\mathbf{K}_{i}(k) \mathbf{C}_{i}(k)\right]^{\mathrm{T}} } \\
& +\mathbf{K}_{i}(k) \mathbf{V}_{i}(k) \mathbf{K}_{i}^{\mathrm{T}}(k)
\end{aligned}
$$

For the master filter

$$
\begin{aligned}
& \widehat{\boldsymbol{x}}_{\mathrm{M}}^{-}(k+1)=\mathbf{A}_{\mathrm{M}}(k) \widehat{\boldsymbol{x}}_{\mathrm{M}}^{+}(k)+\mathbf{B}_{\mathrm{M}}(k) \boldsymbol{u}_{\mathrm{M}}(k) \\
& \mathbf{P}_{\mathrm{M}}^{-}(k+1)=\mathbf{A}_{\mathrm{M}}(k) \mathbf{P}_{\mathrm{M}}^{+}(k) \mathbf{A}_{\mathrm{M}}^{\mathrm{T}}(k)+\mathbf{W}_{\mathrm{M}}(k)
\end{aligned}
$$

For global estimation

$$
\begin{aligned}
& \mathbf{P}_{f}^{+}(k)=\sum_{i=1}^{N}\left[\mathbf{P}_{i}^{+}(k)\right]^{-1}-\left[\mathbf{P}_{\mathrm{M}}^{-}(k)\right]^{-1} \\
& \hat{\boldsymbol{x}}_{f}^{+}(k)=\mathbf{P}_{f}^{+}(k)\left[\mathbf{P}_{M}^{-(k)^{-1}} \hat{\boldsymbol{x}}_{i}^{-}(k)+\sum_{i=1}^{N} \mathbf{P}_{i}^{+}(k)^{-1} \hat{\boldsymbol{x}}_{i}^{+}(k)\right]
\end{aligned}
$$

where $\mathbf{P}_{f}^{+}(k)$ is the inverse of $\mathbf{P}_{f}^{+}(k)^{-1}$.

To detect a sensor fault, an automated checking process is added before the FLA FKF to ensure that the sensor data are sent continuously. In this process, the system verifies the NMEA sentence's header, checksum, and specific characters. An error with any of those causes the system to supply the 
previous measurements instead of the current measurement. If this is persistent, the failed sensors are isolated immediately. This mechanism can simply improve the computation efficiency when the system is working under a large and complex sensor network. On the other hand, a GPS output is requested by the system at the same sampling rate as other sensors. The current GPS data update rate is $1 \mathrm{~Hz}$, which is much slower than any other navigation sensor on the Springer. This is done so that the GPS information not only provides location of the vehicle but also guarantees successful vehicle operation even in the worst-case scenario of the failure of all compasses.

The next section details the formulation of a guidance and control strategy for the Springer vehicle.

\subsection{Guidance and control}

Certain guidance laws will be utilized in the Springer vehicle and integrated with the control system to perform various missions. The simplest of these is the line-of-sight (LOS) guidance which is at the heart of most guidance laws. In this guidance strategy, the vehicle follows the course between any two given points. Another version of the LOS is the waypoint guidance where a number of waypoints are defined between the start and destination coordinates. The next waypoint is selected when the vehicle enters within the circle of acceptance (defined as twice the length of the vehicle) of the current waypoint. A comprehensive review of guidance strategies for unmanned underwater and airborne vehicles has been carried out by Naeem et al. [14]. For environmental monitoring, for instance to detect the source of a chemical discharge, the standard guidance system is replaced. In this case, the USV follows the direction of a chemical plume detected by the onboard sensors and the vessel is guided towards the source of the discharge. The reader is referred to another review paper [15] which is mainly based on chemical plume tracing and odour source localization by autonomous vehicles.

As mentioned earlier, the steering of Springer is based on differential thrust. The vehicle will traverse in a straight line if the speeds of the propellers are the same. Any difference in the propellers' speeds makes the vehicle turn in one direction or the other. To maintain the speed of the vessel at all times, it is important that the average speeds of the propellers are kept the same. The manoeuvres are then obtained by changing the revolution rates of both motors whilst keeping the average speeds constant at all times.

For autopilot design, an LQG controller is selected which consists of a linear combination of a linear quadratic state feedback regulator (LQR) and a Kalman filter. The LQG controller is inherently multivariable; therefore modification to a multiinput multi-output model is rather straightforward. To construct the autopilot, an LQR problem is solved which assumes that all states are available for feedback. However, this is not always true because either there is no available sensor to measure that state or the measurement is very noisy. A Kalman filter can be designed to estimate the unmeasured states. The LQR and Kalman filter are developed independently and then combined to form an LQG controller, a fact known as the separation principle [16]. A block diagram of the controller is depicted in Fig. 12 showing the individual components of the LQG. In this figure, $\boldsymbol{r}$ is the reference input which is transformed to a corresponding reference state vector using the transformation matrix $\mathbf{N}_{x}$. The block $\mathbf{N}_{\boldsymbol{u}}$ compensates for any steady state errors present in the output of the closed-loop system.

The LQG controller requires a state space model of the system in the form specified in equation (4).

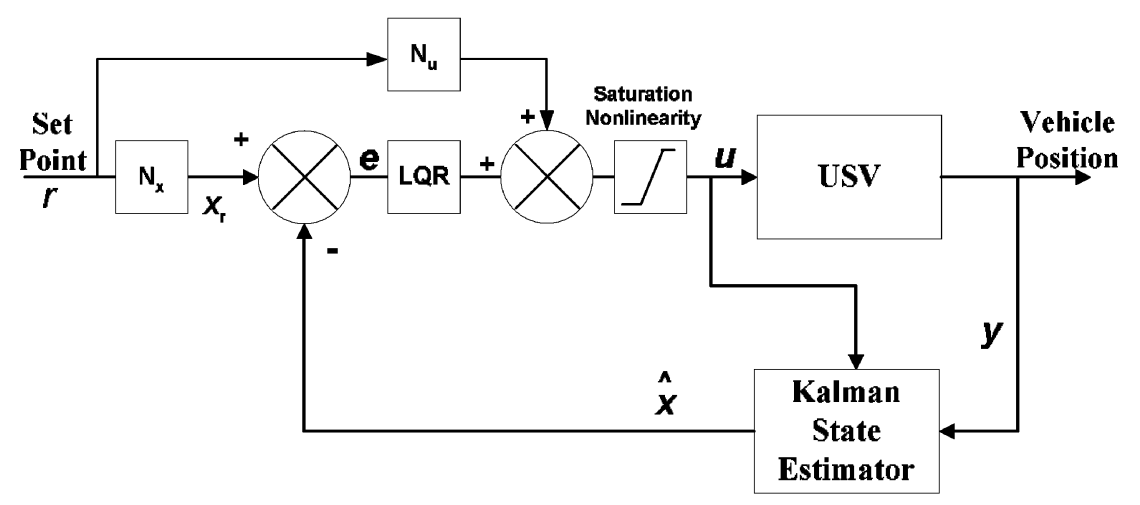

Fig. 12 Block diagram representation of an LQG controller 
Table 2 LQG controller parameters

\begin{tabular}{ll}
\hline Parameter & Value \\
\hline State weighting matrix $\mathbf{Q}$ & 100 \\
Input weighting matrix $\mathbf{R}$ & 0.001 \\
Process noise covariance matrix $\mathbf{W}$ & $10^{-7}$ \\
Measurement noise covariance matrix $\mathbf{V}$ & 1 \\
\hline
\end{tabular}

The parameters $\mathbf{A}, \mathbf{B}, \mathbf{C}$, and $\mathbf{D}$ are also defined in equation (5) for the Springer vehicle. A unique closed-form solution of the LQG control law is defined as

$$
\boldsymbol{u}(k)=\mathbf{K}_{\mathrm{LQR}}\left[\boldsymbol{x}_{\mathrm{r}}(k)-\widehat{\boldsymbol{x}}(k)\right]
$$

See Appendixes 2 and 3 for Kalman filter equations and a derivation of the $\mathbf{N}_{x}$ and $\mathbf{N}_{\boldsymbol{u}}$ blocks respectively.

Herein, the intention is to validate the model of the USV by generating a step response through simulation of a closed-loop control system. It should be noted that most of the missions undertaken by unmanned vehicles are mainly based on following a specified heading angle or following the LOS angle between two waypoints. Hence it is found sufficient to include only a step response of the Springer model to validate the closed-loop system.

The parameters of the LQG autopilot are listed in Table 2 and are selected through a heuristic approach.

The constraints on the differential thrust are assumed to be $\pm 300 \mathrm{r} / \mathrm{min}$ where a positive value represents a starboard side manoeuvre and a negative value corresponds to a port side movement. The simulated response is depicted in Fig. 13(a) showing the reference output, the noisy sensor

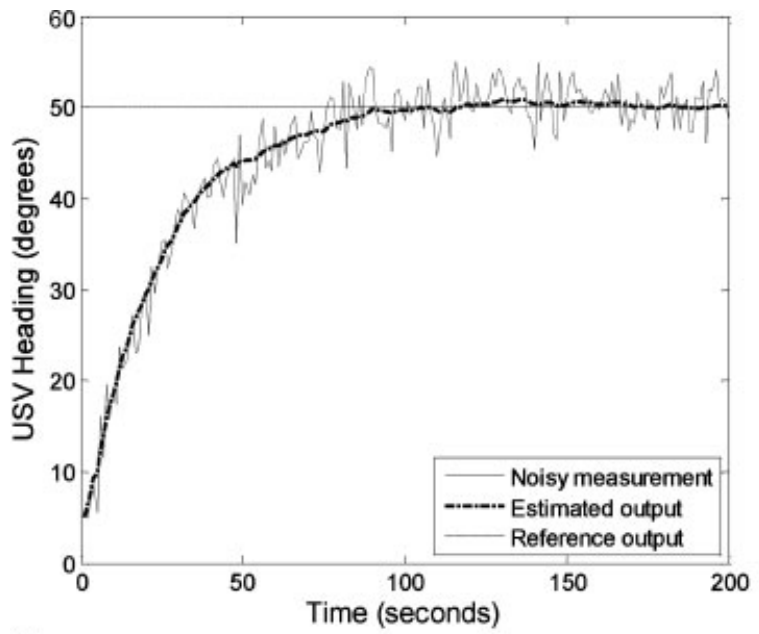

(a) measurement, and the estimated output of the Kalman filter. The simulated noise added to the system is mainly the high-frequency sensor noise. Any drifts or disturbances due to winds or currents are not considered in this paper. The initial vehicle orientation is $10^{\circ}$ with respect to the north and it takes approximately $90 \mathrm{~s}$ for the vehicle to reach the desired heading angle of $50^{\circ}$. This corresponds to a starboard side manoeuvre throughout the simulation which should also be reflected in the controller output. Figure 13(b) shows the differential input $n_{\mathrm{d}}$ to the system which gradually settles to zero from a positive initial value. The figure shows the minimum control effort as the bulk of the noise portion is processed by the Kalman filter which removes the undesirable oscillations in the control input.

\section{CONCLUSIONS}

Although industrialization has its own obvious benefits, it has had a devastating impact on the environment mainly owing to the toxic nature of waste produced in the process. It is vital that its disposal should be monitored and dealt with in a proper way in order to reduce its effect on the sustainability of the environment. In view of this, a new USV named Springer is being developed to carry out environmental data gathering and pollutant tracking. This paper highlights up-to-date NGC hardware and software developments on the Springer USV including trials results and modelling using SI techniques. The USV was tested in open water numerous times and several useful observations were made which aided the modelling and

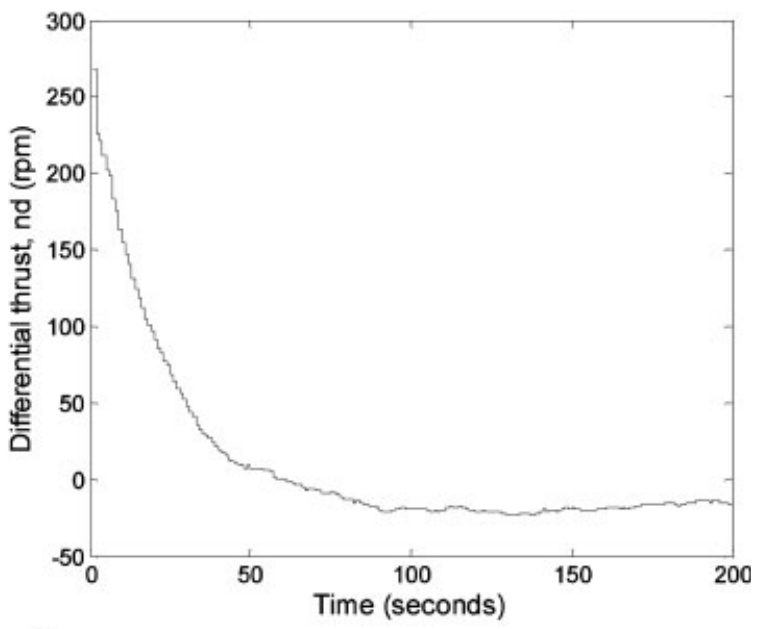

(b)

Fig. 13 (a) Reference input tracking of an LQG autopilot showing the noisy measurement and filtered output. (b) Differential thrust to the USV generated by the LQG autopilot 
control system design. An LQG autopilot is formulated and simulated for the identified model. Results are shown which clearly validate the suitability of the model and the autopilot. Furthermore, a novel form of MSDF algorithm based on soft computing methodology is proposed. The next step is to implement the integrated NGC system in real time and to assess the autonomous performance of the vessel in full-scale sea trials.

\section{ACKNOWLEDGEMENT}

The authors wish to acknowledge the Engineering and Physical Sciences Research Council for funding this project.

\section{REFERENCES}

1 Naeem, W., Xu, T., Chudley, J., and Sutton, R. Design of an unmanned surface vehicle for environmental monitoring. In Proceedings of the World Maritime Technology Conference, London, UK, March 2006.

2 Corfield, S. J. Unmanned surface vehicles and other things. In Proceedings of the Unmanned Underwater Vehicle Showcase 2002 Conference, Southampton, UK, 2002, pp. 83-91.

3 Young, H. W. and Phillips, S. J. Development of an autonomous semi-submersible for deploying sensors for ocean survey. In Proceedings of the International UUV Symposium, Newport, Rhode Island, USA, 2000.

4 Reed, C. M., Bishop, B. E., and Waters, J. K. Design of an autonomous surface vessel. In Proceedings of the World Maritime Technology Conference, London, UK, March 2006.

5 Hook, D. J. Development of unmanned surface vehicles. In Proceedings of the World Maritime Technology Conference, London, UK, March 2006.

6 Naeem, W., Xu, T., Sutton, R., and Chudley, J. Design of an unmanned catamaran with pollutant tracking and surveying capabilities. In Proceedings of the UKACC International Control Conference, IMechE Mini Symposia, Glasgow, Scotland, UK, August 2006, pp. 99-113 (Institution of Engineering and Technology, London).

7 Xu, T., Chudley, J., and Sutton, R. A fuzzy logic based multi-sensor navigation system for an unmanned surface vehicle. In Proceedings of the UKACC International Control Conference, Glasgow, UK, August 2006.

8 YSI environmental operations manual, available from http://www.YSI.com, date accessed, 18 October 2005.

9 RoboteQ AX2550/2850 user's manual, available from http://www.roboteq.com, date accessed, 18 October 2005.

10 Naeem, W. Guidance and control of an autonomous underwater vehicle. PhD Thesis, School of Engineering, University of Plymouth, 2004.

11 Ljung, L. System identification, theory for the user, 2nd edition, 1999 (Prentice-Hall PTR, Englewood Cliffs, New Jersey).

12 Farrell, A. J. and Barth, M. The global positioning system and inertial navigation, 1999 (McGraw-Hill, New York).

13 Mehra, R. On the identification of variances and adaptive Kalman filtering. IEEE Trans. Autom. Control, 1970, 15(2), 175-184.

14 Naeem, W., Sutton, R., Ahmad, S. M., and Burns, R. S. A review of guidance laws applicable to unmanned underwater vehicles. J. Navig., 2003, 56(1), 15-29.

15 Naeem, W., Sutton, R., and Chudley, J. Chemical plume tracing and odour source localisation by autonomous vehicles. J. Navig., 2007, 60(2), 173-190.

16 Burl, J. B. Linear optimal control, $H 2$ and $H_{\infty}$ methods, 1999 (Addison-Wesley Longman, Reading, Massachusetts).

17 Franklin, G. F., Powell, J. D., and Workman, M. Digital control of dynamic systems, 3rd edition, 1998 (Addison-Wesley Longman, Reading, Massachusetts).

\section{APPENDIX 1}

\section{Notation}

$d$

K

$\mathbf{K}_{\mathrm{LQR}}$

$n_{\mathrm{c}}$

$n_{\mathrm{d}}$

$n_{1}, n_{2}$

$\mathbf{N}_{u}$

$\mathbf{N}_{x}$

$\mathbf{P}$

$\boldsymbol{u}$

$v$

V

$w$

W

$\boldsymbol{x}$

$\boldsymbol{x}_{\mathrm{r}}$

$\boldsymbol{x}_{\mathrm{ss}}$

$\hat{\boldsymbol{x}}$

$y$

$\hat{\boldsymbol{y}}$

$\beta$ disturbance input

Kalman filter gain

LQR gain of the controller

common mode thruster velocity

( $\mathrm{r} / \mathrm{min})$

differential mode thruster velocity

$(\mathrm{r} / \mathrm{min})$

thruster velocities of individual

propellers

steady state error compensator

reference state transformation matrix error covariance matrix

input to the system

measurement noise

measurement noise covariance

matrix

process noise

process noise covariance matrix

state vector of the process

reference state vector

system state vector at steady state

estimated state vector

output of the system

predicted output from the model

adaptive feedback factor of the proposed fuzzy multi-sensor data fusion algorithm 


\section{APPENDIX 2}

Given a discrete-time controlled process described by the linear stochastic difference equations

$$
\begin{aligned}
& \boldsymbol{x}(k+1)=\mathbf{A} \boldsymbol{x}(k)+\mathbf{B} \boldsymbol{u}(k)+\boldsymbol{w}(k) \\
& \boldsymbol{z}(k)=\mathbf{C} \boldsymbol{x}(k)+\mathbf{D} \boldsymbol{u}(k)+\boldsymbol{v}(k)
\end{aligned}
$$

where $\boldsymbol{x}(k)$ is an $n \times 1$ state vector, $\mathbf{A}$ is an $n \times n$ state transition matrix, $\boldsymbol{u}(k)$ is an $l \times 1$ input vector, $\mathbf{B}$ is an $n \times 1$ matrix, $\boldsymbol{w}(k)$ is an $n \times 1$ process noise vector, $z(k)$ is an $m \times 1$ measurement vector, $\mathbf{C}(k)$ is an $m \times$ $n$ measurement matrix, and $\boldsymbol{v}(k)$ is an $m \times 1$ measurement noise vector. Both $\boldsymbol{w}(k)$ and $\boldsymbol{v}(k)$ are assumed to be uncorrelated zero mean Gaussian white-noise sequences with covariances given by

$$
\begin{aligned}
& E\left[\boldsymbol{w}(k) \boldsymbol{w}^{\mathrm{T}}(i)\right]=\left\{\begin{array}{cc}
\mathbf{W}(k), & i=k \\
0, & i \neq k
\end{array}\right. \\
& E\left[\boldsymbol{v}(k) \boldsymbol{v}^{\mathrm{T}}(i)\right]=\left\{\begin{array}{cc}
\mathbf{V}(k), & i=k \\
0, & i \neq k
\end{array}\right. \\
& E\left[\boldsymbol{w}(k) \boldsymbol{v}^{\mathrm{T}}(i)\right]=0, \quad \text { for all } k \text { and } i
\end{aligned}
$$

The Kalman filter equations can be written into the time update equations

$$
\begin{aligned}
& \widehat{\boldsymbol{x}}(k+1)=\mathbf{A} \widehat{\boldsymbol{x}}(k)+\mathbf{B} \boldsymbol{u}(k) \\
& \mathbf{P}^{-}(k+1)=\mathbf{A} \mathbf{P}(k) \mathbf{A}^{\mathrm{T}}+\mathbf{W}(k)
\end{aligned}
$$

and the measurement update equations

$$
\begin{aligned}
& \mathbf{K}(k)=\mathbf{P}^{-}(k) \mathbf{C}^{\mathrm{T}}\left[\mathbf{C} \mathbf{P}^{-}(k) \mathbf{C}^{\mathrm{T}}+\mathbf{V}(k)\right]^{-1} \\
& \widehat{\boldsymbol{x}}(k)=\widehat{\boldsymbol{x}}^{-}(k)+\mathbf{K}(k)\left[\boldsymbol{z}(k)-\mathbf{C} \widehat{\boldsymbol{x}}^{-}(k)\right] \\
& \mathbf{P}(k)=[\mathbf{I}-\mathbf{K}(k) \mathbf{C}] \mathbf{P}^{-}(k)
\end{aligned}
$$

The measurement update equations incorporate a new observation into the a priori estimate from the time update equations to obtain an improved $a$ posteriori estimate. In the time and measurement update equations, $\hat{\boldsymbol{x}}$ is an estimate of the system state vector $\boldsymbol{x}, \mathbf{K}$ is the Kalman gain, and $\mathbf{P}$ is the covariance matrix of the state estimation error.

\section{APPENDIX 3}

A derivation has been carried out herein to evaluate the contents of blocks $\mathbf{N}_{\boldsymbol{x}}$ and $\mathbf{N}_{\boldsymbol{u}}$ in Fig. 12 for reference input tracking in an LQR control strategy [17].

Let $\boldsymbol{N}_{x}$ denote the forward block which transforms the reference input $\boldsymbol{r}$ to a reference state $\boldsymbol{x}_{\mathrm{r}}$ i.e. an equilibrium state for that $\boldsymbol{r}$. Mathematically, this can be stated as

$$
\begin{aligned}
& \mathbf{N}_{x} r=x_{\mathbf{r}} \\
& \boldsymbol{u}=-\mathbf{K}_{\mathrm{LQR}}\left(\boldsymbol{x}-\boldsymbol{x}_{\mathbf{r}}\right)
\end{aligned}
$$

The final or steady state value of the states can be written as

$$
\boldsymbol{x}(\infty)=\boldsymbol{x}_{\mathrm{ss}}=\boldsymbol{x}_{\mathrm{r}} \Rightarrow \mathbf{N}_{x} r=\boldsymbol{x}_{\mathrm{r}}=\boldsymbol{x}_{\mathrm{ss}}
$$

To compensate for any steady state output error in the case of type 0 systems, a steady state control term is needed that is proportional to the reference input according to

$$
\boldsymbol{u}_{\mathrm{ss}}=\mathbf{N}_{u} r
$$

Also

$$
\mathrm{Cx}_{\mathrm{ss}}=\boldsymbol{y}=\boldsymbol{r} \Rightarrow \mathrm{CN}_{x} r=\boldsymbol{r} \Rightarrow \mathrm{CN}_{x}=\mathrm{I}
$$

Since the system is at steady state, i.e. $\boldsymbol{x}(k+1)=\boldsymbol{x}(k)$ $=\boldsymbol{x}_{\mathrm{ss}}$ and $\boldsymbol{u}=\boldsymbol{u}_{\mathrm{ss}}$, therefore

$$
\boldsymbol{x}_{\mathrm{ss}}=\mathbf{A} \boldsymbol{x}_{\mathrm{ss}}+\mathbf{B} \boldsymbol{u}_{\mathrm{ss}} \Rightarrow(\mathbf{A}-\mathbf{I}) x_{\mathrm{ss}}+\mathbf{B} \boldsymbol{u}_{\mathrm{ss}}=0
$$

Substituting equations (30) and (31) in the above equations gives

$$
(\mathbf{A}-\mathbf{I}) \mathbf{N}_{x} r+\mathbf{B} \mathbf{N}_{u} r=0 \Rightarrow(\mathbf{A}-\mathbf{I}) \mathbf{N}_{x}+\mathbf{B N}_{u}=0
$$

Finally writing equations (31) and (33) in matrix form gives

$$
\left[\begin{array}{cc}
\mathbf{A}-\mathbf{I} & \mathbf{B} \\
\mathbf{C} & \mathbf{0}
\end{array}\right]\left[\begin{array}{l}
\mathbf{N}_{x} \\
\mathbf{N}_{u}
\end{array}\right]=\left[\begin{array}{l}
\mathbf{0} \\
\mathbf{I}
\end{array}\right]
$$

and solving for $\mathbf{N}_{x}$ and $\mathbf{N}_{\boldsymbol{u}}$ yields the desired result

$$
\left[\begin{array}{l}
\mathbf{N}_{x} \\
\mathbf{N}_{u}
\end{array}\right]=\left[\begin{array}{cc}
\mathbf{A}-\mathbf{I} & \mathbf{B} \\
\mathbf{C} & \mathbf{0}
\end{array}\right]^{-1}\left[\begin{array}{l}
\mathbf{0} \\
\mathbf{I}
\end{array}\right]
$$

